\title{
Post-Newtonian properties of EMRI with power law potential
}

\author{
Chinmay N. Gandevikar ${ }^{1, a}$, Divyesh N. Solanki ${ }^{2, b}$, Dipanjan Dey ${ }^{3, \mathrm{c}} \mathbb{D}$ \\ ${ }^{1}$ BITS Pilani K.K. Birla Goa Campus, Sancoale, Goa 403726, India \\ ${ }^{2}$ Sardar Vallabhbhai National Institute of Technology, Surat, Gujarat 395007, India \\ ${ }^{3}$ International Center for Cosmology, Charusat University, Anand, Gujarat 388421, India
}

Received: 5 March 2021 / Accepted: 25 July 2021 / Published online: 4 August 2021

(C) The Author(s) 2021

\begin{abstract}
There are many astrophysical scenarios where extreme mass ratio inspiral (EMRI) binaries can be surrounded by inhomogenous matter distribution. The distribution of mass can affect the dynamical properties (e.g. orbital frequency, average energy radiation rate, etc.) of the EMRI. In this matter distribution, instead of Kepler-Newton potential, one may consider a more general form of potential i.e. power law potential. Moreover, due to the power law potential, at the Newtonian order itself, the velocity profile of test particles does not fall as much as that predicted by Kepler-Newton potential and this feature of the velocity profile may be observationally important. In this study, we have obtained the first post-Newtonian (1PN) expressions for dynamical quantities and the average energy radiation rate from the circular orbit EMRI which is surrounded by a matter distribution. We show that the energy radiation rate and orbital frequency of EMRI can be significantly different in the presence of power law potential as compared to that in the Kepler-Newton potential, signatures of which may be observed in gravitational waves from EMRI.
\end{abstract}

\section{Introduction}

In a galaxy, the study of dynamics of the bodies around the central object has been of great interest for the last four decades. According to Kepler-Newton potential, the velocities of the stars about a central massive/supermassive object should drop drastically with increasing radial distance from the central object. However, observations show otherwise. The Kepler-Newton expression (also denoted as KN potential) of gravitational potential $\left(\frac{G m}{r}\right)$ known to us can be obtained as the weak field approximate of the effective potential of the Schwarzschild spacetime very far away from

\footnotetext{
a e-mail: cgandevikar@gmail.com

b e-mail: divyeshsolanki98@gmail.com

c e-mail: deydipanjan7@gmail.com (corresponding author)
}

the central body. However, the Schwarzschild metric is a vacuum solution of the Einstein's field equation. But at the galactic scale, due to dark matter and baryonic matter distribution, the system is non-vacuum. Due to this limitation, the Schwarzschild metric or the KN potential cannot theoretically explain galactic dynamics.

In the late 1970s and early 1980s, it was confirmed by the extensive study reported in Sofue and Rubin in 2001 [1] that most of the stars in a galaxy rotate at nearly the same velocities. This means that the velocities of these stars are independent of their distance from the central mass. Investigation of this novel observation has been a hot topic for several decades. There have been multiple theories like modified Newtonian dynamic (MOND) [2], the existence of dark matter, modified gravity, etc. [3-5]. In 1933, Zwicky [6] suggested "missing mass" to account for the orbital velocities of galaxies in clusters. Later, V Rubin and their group studied the possibility of dark matter around galaxies [7,8]. In [9, 10], authors study the mass density distribution in galaxies and compare them with the observations. The density profile of dark matter famously known as the Navvaro-Frenk-White (NFW) profile [11] in the galaxy justifies the nature of the curve. Dey et al. have worked on developing a general relativistic approach to understand galactic dynamics. Bertrand Spacetimes is one of the non-vacuum spacetime which can be thought to be seeded by dark matter [12-15]. In the Newtonian limit, this spacetime can effectively give the NFW dark matter density profile and hence can explain the observed behaviour of rotational velocity curves. In 1983, Milgrom suggested modifying Newtonian dynamics (MOND) which is an alternate way to justify the curves. According to the MOND, Newton's laws of gravity get modified at a large distance scale. The theoretical prediction of MOND satisfactorily fit the observed velocity profiles of stars for a large number of galaxies [16]. Later, relativistic study of MOND was done by Bekenstein [17], Moffat [18,19] and Brownstein 
[20]. So there are many ways to look at the mismatch of the velocity profiles.

In our study we consider that an appropriate power law potential is obtained from a particular mass distribution (inhomogenous) in the region. This potential can lead to the rotational velocities (matching the observations) higher than that in the case of $\mathrm{KN}$ potential. The velocity curve plotted using the logarithmic potential is completely flat. Recently, Munera and Delgado-Correal [21] have shown derivation of logarithmic potential as a solution of non-homogeneous Laplace equation. Power law forces are mentioned in classical books like Goldstein [22] and Danby [23]. The power law potentials (also denoted as PL potential) have been extensively studied in the non-relativistic case. In [24-26], the nature of orbits in the presence of power law potential is studied extensively. Here, we look at the problem in this way (considering only general relativity and Newtonian mechanics) rather than in the direction of modified gravity as used in MOND [2].

In a scenario where there is only a point mass with vacuum around it, the $\mathrm{KN}$ potential can be obtained as an approximate potential derived from the Schwarzschild solution of Einstein field equations (in a region which is far away from the source). The dynamics of such a system has been extensively studied and has been documented very well in classical textbooks [22,27]. For a point mass binary, in their seminal work, Peters and Mathews have derived the binary dynamics and the gravitational radiation [28]. Extreme mass-ratio inspirals (EMRIs) are expected to be a significant source of gravitational waves that can be detected in the milli-hertz band (by upcoming space-based detector LISA) [29-32]. In the past decade there have been many works on EMRIs discussing their astrophysical population [33], their orbital evolution [34], their detectability based on their position and orientation [32-34], the waveforms of radiation from them [35] etc. The considered EMRIs usually constitute a massive/supermassive compact object and a stellar mass object in vacuum environment.

In our paper we consider a mass distribution around the massive/supermassive object and we report the dynamics and the average energy radiation rate from toy model circularorbit-EMRI where the two bodies are very far apart. Considering only two point particles is not entirely directly applicable to study binary systems in galaxies or systems with dark matter distribution around them. One may choose to sum-over the effects of KN potential due to every particle to encompass the effects due to the mass distribution. In this paper we consider a more general power law potential that can (as shown in Sect. 1) incorporate the effects of this mass distribution and whose density falls radially away from a point (where the potential is undefined and the mass density is infinite). This mass density also can justify the behaviour of the velocity profile. In this paper, we consider an extreme mass ratio inspiral (EMRI) binary of a stellar mass object which is orbiting a massive/supermassive object. The mass distribution around this massive/supermassive object and the potential due to it both have a power law form. The orbital motion makes the EMRI radiate energy which propagates as the gravitational waves. We consider that this stellar object is in the faraway region from the central massive/supermassive object. This region allows the usability of Post-Newtonian (PN) theory to obtain more precise expressions for the quantities that can be described for the system, e.g., orbital velocity of the object, the orbital radius, the acceleration of the object etc. Subsequently, we predict the average energy radiation rate from the system. The observational aspects of such systems, higher order of PN corrections, orbital evolution and consideration of highly eccentric orbits are out of scope of this paper and will be discussed in upcoming papers.

We organize the paper in the following way. In Sect. 2, we introduce an inhomogenous matter density $\left(\rho\left(r^{\prime}\right)\right)$ (which has a power law dependence over radial distance from the center of mass) for a massive/supermassive compact object. We then show that the gravitational potential due to the matter in this distribution has a power law form. In Sect. 3, we derive the acceleration of a body up to 1PN approximation using power law potential instead of $\mathrm{KN}$ potential. The binary system and the PN corrected expressions for the dynamical quantities are discussed in Sect. 4. In Sect. 5, we discuss the PN corrected average energy radiation rate. In Sect. 6, the significant dynamical variables like the velocity and the orbital frequency, and energy radiation in the case of power law potential are compared with that in the case of KN potential system. In Sect. 7, we discuss the important results and possible future works.

\section{Matter distribution and resultant potential}

Let's consider an inhomogenous matter distribution around a singularity (like that inside a black hole) or point where the mass density is indefinitely high. This object can be a massive/supermassive compact object. We call this object as body-Q. Let the mass density profile $\left(\rho\left(r^{\prime}\right)\right)$ of and around body-Q (here $r^{\prime}$ is the distance from the center of mass) be given as,

$\rho\left(r^{\prime}\right)=\frac{1-2 \delta}{2 \delta} \frac{1}{4 \pi} M_{Q}^{*} \frac{1}{r^{\prime 2 \delta+2}}$,

where, $M_{O}^{*}=M_{Q} \times \epsilon^{2 \delta-1}$ is a constant scale mass and $\epsilon$ is the scale size of the object-Q, and $\delta$ is an arbitrary constant (with value $<1 / 2$ ). It should be noted that we consider a coordinate system with its origin at the center of mass of the massive/supermassive object. Also if we substitute $\delta=1 / 2$ we will get a Delta function (the $1-2 \delta$ makes the numerator $=0$ and the denominator becomes $=0$ only when $r^{\prime}=0$, 
i.e., the density is undefined at $r^{\prime}=0$ and is zero everywhere else) like the one used for Newtonian dynamics.

The potential experienced by a body-P which is at $r$ distance from the body-Q due to a small mass element at $r^{\prime}$ distance can be calculated. Now this effect can be easily integrated over the surface of the sphere, which leads to the potential due to a shell of thickness $d r^{\prime}$ (centered at the center of mass of the matter distribution i.e., the origin of the coordinate system) at a test mass body-P which is $r$ distance away from the center of mass of the matter distribution is given as,

$$
\begin{aligned}
d U_{P} & =\frac{G}{r} d m \\
& =\frac{4 \pi G}{r} \rho\left(r^{\prime}\right) r^{\prime 2} d r^{\prime}
\end{aligned}
$$

Integrating this expression to the whole volume till body $\mathrm{P}$, we get the total potential at $\mathrm{P}$ due to the whole mass distribution,

$$
\begin{aligned}
U_{P}=\int_{U_{\infty}}^{U_{P}} d U_{P, \text { Shell }} & =\int_{\infty}^{r} \frac{4 \pi G}{r} \rho\left(r^{\prime}\right) r^{\prime 2} d r^{\prime} \\
\Longrightarrow U_{P} & =\frac{G}{2 \delta r^{2 \delta}} M_{Q}^{*} .
\end{aligned}
$$

To obtain the second line of the above equation we have substituted the value of $\rho\left(r^{\prime}\right)$ from (Eq. 1). This expression can be verified by noting that if $\delta=1 / 2$ we would get the Kepler Newton potential i.e., $U_{K N}=\frac{G M_{Q}}{r}$ which would be an effect of a mass density which has a delta function form. The Newtonian order dynamical effects of this form of potential have been discussed in [37-39]. This potential can be used to justify the observed velocity profile of stellar mass objects around massive/supermassive compact objects. Note:

1. We assume that the mass of body-P is much smaller than the mass of body- $\mathrm{Q}$ and the mass distribution is concentrated at the center of mass and then drops sharply with increasing $r^{\prime}$. Which means that the distance between the two bodies is much larger than the scale length of the massive/supermassive body-Q. This means that the gravitational effects of body-P are negligible on the mass distribution around Q. For simplicity of calculations in this paper, we assume that the motion of any matter element in this distribution is negligible.

2. In this paper, the obtained potential is a result of the (classical-Newtonian) gravitational effects of the matter distribution and the derivation of the same does not involve any other theory of gravity.

\section{Acceleration of the reference body in the presence of power law potential}

We have established the fact that the power law potential is an effect of sum of all the infinitesimally small mass elements near the point-Q. At Newtonian order, for KN potential, the acceleration is given by $a_{P, K N}^{j}=\partial^{j} U_{P, K N}$. Now in the case of power law potential, the acceleration can be obtained by adding the acceleration due the infinitesimal potential due to an infinitesimal element of the mass in the distribution. Hence the Newtonian order acceleration $\left(a_{P, N}^{j}\right)$ (the subscript $\mathrm{P}$ denotes the body-P, $\mathrm{N}$ denotes the Newtonian order and $\mathrm{KN}$ denotes Kepler-Newton potential),

$$
\begin{aligned}
a_{P, N}^{j} & =\int d a_{P, N, K N}^{j}=\int d\left(\partial^{j} U_{P, K N}\right) \\
& =\partial^{j} \int\left(d U_{P, K N}\right)=\partial^{j} U_{P}
\end{aligned}
$$

The first post-Newtonian order corrected approximate expression for acceleration vector $\left(a_{P}^{j}\right)$ of a body named as body-P as derived in [36] is valid in our study. The acceleration is given as,

$$
\begin{aligned}
a_{P}^{j}= & \partial^{j} U_{P}+\frac{1}{c^{2}}\left[\left(v_{P}^{2}-4 U_{P}\right) \partial^{j} U_{P}-4 v_{P}^{j} v_{P}^{k} \partial_{k} U_{P}\right. \\
& -3 v_{P}^{j} \dot{U}_{P}+\partial^{j} \psi_{P}+\frac{1}{2} \partial^{j} \ddot{X}_{P}+4 \dot{U}_{P}^{j} \\
& \left.-4\left(\partial^{j} U_{P}^{k}-\partial^{k} U_{P}^{j}\right) v_{P, k}\right] \\
& +O\left(c^{-4}\right),
\end{aligned}
$$

where $U_{P}, U_{P}^{j}, \psi_{P}^{j}$ and $X_{P}$ are the external scalar gravitational potential, vector gravitational potential, postNewtonian correction to scalar gravitational potential and the super-potential respectively.

The components of the EMRI under consideration are a massive/supermassive compact object- $Q$ of mass $M_{Q}$ and a stellar mass object- $P$ of mass $M_{P}$ (which is much less than that of body- $Q$ ). We use the following notations throughout the paper: $\mathbf{y}_{Q}$ and $\mathbf{y}_{P}$ are the position vectors of the bodies $\mathrm{Q}$ and $\mathrm{P}$ in the center of mass frame, $r_{P Q}$ is the distance between the two bodies, also given as $\left|\mathbf{y}_{P}-\mathbf{y}_{Q}\right| \cdot \mathbf{v}_{Q}$ and $\mathbf{v}_{P}$ are the absolute velocities of the bodies, $\mathbf{a}_{Q}$ and $\mathbf{a}_{P}$ are the absolute accelerations of the bodies, $\mathbf{x}_{S}$ is the position vector of any field point ' $S$ ' where potential is being evaluated. The unit vector in $\mathbf{x}_{S Q}$ direction and the unit vector in the direction of $\mathbf{x}_{P Q}$ can be written as $\hat{\mathbf{n}}_{S Q}=\frac{\mathbf{x}_{\mathbf{S Q}}}{\left|\mathbf{x}_{\mathbf{S Q}}\right|}=\frac{\mathbf{x}_{S}-\mathbf{y}_{Q}}{\left|\mathbf{x}_{S}-\mathbf{y}_{Q}\right|}$ and $\hat{\mathbf{n}}_{P Q}=\frac{\mathbf{x P Q}_{\mathbf{P Q}}}{|\mathbf{X P Q}|}=\frac{\mathbf{y}_{P}-\mathbf{y}_{Q}}{\left|\mathbf{y}_{P}-\mathbf{y}_{Q}\right|}$ respectively.

With increasing radial distance from the central massive/supermassive object, according to Kepler's laws, the rotational velocities are expected to fall down to nearly zero. However the observations show otherwise. The velocity curves do not drop as much as predicted. In order to have higher values of rotational velocities (which is gener- 
ally observed in galactic scales $[1,7,8])$, one can use specific forms of power law potential (Eq. 3) [37,38]. A general form of the expression of the Newtonian order (i.e. 0 PN order) gravitational acceleration $\mathbf{a}_{P, N}(r)$ for the power law potential can be written as,

$\mathbf{a}_{P, N}\left(r_{P Q}\right)=\partial^{j} U_{P}\left(r_{P Q}\right)=-\frac{G M_{Q}^{*} r_{P Q}}{\left(r_{P Q}^{2}\right)^{\delta+1}} \hat{\mathbf{n}}_{P Q}$.

Here, $M_{Q}^{*}=M(\epsilon) \epsilon^{2 \delta-1}$ is the constant scale mass of the potential due to body-Q, $\epsilon$ is the scale radius of the source and $\delta$ can have any real value [39]. Note that the subscript in the acceleration is " $P, N$ " denoting acceleration of body$\mathrm{P}$ at Newtonian order, which is different from " $P N$ " which denotes the post-Newtonian corrections. Two interesting special cases are

1. The circular velocity is constant $\left(v_{\text {circ }}=\sqrt{|\mathbf{a}| r}=\right.$ const $)$ i.e. $\delta=0$ and

2. The circular velocity is inversely proportional to the square root of the radial distance $\left(v_{\text {circ }}=\sqrt{|\mathbf{a}| r} \propto\right.$ $\left.r^{-1 / 2}\right)$ i.e. $\delta=\frac{1}{2}$.

Above two cases correspond to flat and Keplerian rotation curves, respectively. The exactly flat rotation curves can be obtained at $\delta=0$. Using Eq. (6), it can be seen that the corresponding potential is the logarithmic potential. In this study, the general power law potential is taken under consideration except for the logarithmic potential case.

\subsection{External potentials and their derivatives}

In this sub-section, we derive the post-Newtonian potentials and their derivatives as introduced in Eq. (5). It is considered that the distance between the two bodies is much larger than the sizes of the two bodies. From here on, external potentials are referred to as just "potentials".

Before introducing other external potentials, we introduce the following tools which are used to obtain the same

$$
\begin{aligned}
\partial^{j} r_{S Q} & =n_{S Q}^{j}, \\
\partial_{j} \mathbf{x}_{S Q}^{k} & =\delta_{j}^{k}, \\
\dot{\mathbf{x}}_{S Q} & =-\mathbf{v}_{Q}, \\
\dot{r}_{S Q} & =-\hat{\mathbf{n}}_{S Q} \cdot \mathbf{v}_{Q}, \\
\partial^{j} n_{S Q}^{k} & =\frac{\delta^{j k}}{r_{S Q}}-\frac{n_{S Q}^{j} n_{S Q}^{k}}{r_{S Q}}, \\
\partial^{j} v_{Q}^{k} & =0 .
\end{aligned}
$$

The over-dot represents the time derivative of the quantity. The vector potential $\left(U_{P}^{j}\right)$ corresponding to the gravitational potential (Eq. 3) can be written as [36]
$U_{P}^{j}=\frac{G M_{Q}^{*}}{2 \delta\left(r_{P Q}^{2}\right)^{\delta}} v_{Q}^{j}$

Post-Newtonian correction $\left(\psi_{P}\right)$ to the Newtonian Potential.

$\psi_{P}=U_{P}\left(\frac{3}{2} v_{Q}^{2}-U_{Q}\right)$

where $U_{Q}$ is the potential at body-Q.

Mathematically, potential is the quantity obtained from Poisson's equation using a source term. We need mass density to get the gravitational potential $(U)$. Using this Newtonian gravitational potential as source, a higher order of potential called super-potential $(X)$ can be obtained. In other words super-potential is the potential generated by external potential. Here we will briefly explain the derivation:

The Poisson's equation for super-potential $(X)$ is given as

$\nabla^{2} X=2 U$

The source term contains the potential $U$. The potential due to a mass- $Q$ at a field point $\left(\mathbf{x}^{\prime}\right)$ is a function of the distance between the field point and the source mass is

$U\left(\mathbf{x}^{\prime}\right)=U\left(\mathbf{x}^{\prime}-\mathbf{y}_{Q}\right)$.

The corresponding super-potential $\left(X\left(\mathbf{x}_{S}\right)\right)$ at another field point $\left(\mathbf{x}_{S}\right)$ due to the potential $U\left(\mathbf{x}^{\prime}\right)$ is the solution of the Poisson's equation, $\nabla^{2} X\left(\mathbf{x}_{S}\right)=2 U\left(\mathbf{x}_{S}-\mathbf{x}^{\prime}\right) . X\left(\mathbf{x}_{S}\right)$ is spherically symmetric hence it's dependence on vector $\left(\mathbf{x}_{S}-\mathbf{x}^{\prime}\right)$ can be simplified into the dependence over the magnitude of this vector, which we denote as ' $r$ '. The derivative of this super-potential as required in Eq. (5) is formalized as

$$
\begin{aligned}
\partial^{j} \ddot{X}\left(\mathbf{x}_{\mathbf{S}}\right)= & X^{\prime \prime \prime}(r) \dot{r}^{2} \partial^{j} r+2 X_{P}^{\prime \prime}(r) \dot{r} \partial^{j} \dot{r}+X^{\prime \prime}(r) \ddot{r} \partial^{j} r \\
& +X^{\prime}(r) \partial^{j} \ddot{r} .
\end{aligned}
$$

Here, the primes denote radial derivative and the over-dots are for time derivatives. The general solution to the Poisson's equation is given as

$X\left(\mathbf{x}_{S}\right)=-\frac{1}{2 \pi} \int \frac{U\left(\mathbf{x}^{\prime}\right)}{\left|\mathbf{x}_{S}-\mathbf{x}^{\prime}\right|} d^{3} x^{\prime}+X_{0}\left(\mathbf{x}_{S}\right)$.

Here, $X_{0}$ is the constant of integral, which is the solution to the Laplace equation $\nabla^{2} X_{0}=0$. As prescribed [36] (in the box 7.3) the domain of integration is truncated to spatial near zone. This changes the form of Poisson's equation to $\nabla^{2} X=2 U \Theta(R-r)$, where $\Theta(R-r)$ is the Heaviside step function and $R$ is the radius of the boundary of the near zone (value of which is immaterial and this fact will be used later).

According to our assumption, the body- $\mathrm{Q}$ is heavy enough compared to the body-P such that the effect due to the body- 
$\mathrm{P}$ is negligible as compared to that due to body- $\mathrm{Q}$, and we consider only the effect due to body-Q as

$U\left(\mathbf{x}^{\prime}\right) \approx U\left(\mathbf{x}^{\prime}-\mathbf{y}_{Q}\right)=\frac{G M_{Q}^{*}}{2 \delta\left(r_{1 Q}^{2}\right)^{\delta}}$,

where, $r_{1 Q}=\left|\mathbf{x}^{\prime}-\mathbf{y}_{Q}\right|$.

Substituting Eq. (19) into Eq. (18), we obtain

$X\left(\mathbf{x}_{S}\right)=-\frac{G M_{Q}^{*}}{4 \pi \delta} \int \frac{\Theta\left(R-r^{\prime}\right)}{\left|\mathbf{x}_{S}-\mathbf{x}^{\prime}\right|\left(r_{1 Q}^{2}\right)^{\delta}} d^{3} x^{\prime}+X_{0}\left(\mathbf{x}_{S}\right)$.

From here on, $r^{\prime}=\left|\mathbf{x}^{\prime}\right|$. For the mathematical simplicity, we take $\mathbf{y}_{Q}=0$.

At the end of this section, we generalize our result by introducing $\mathbf{y}_{Q}$. We set the Cartesian coordinates such that the $\mathrm{Z}$-axis aligns with the position vector $\mathbf{x}_{\mathbf{S}}$.

Therefore, $\mathbf{x}_{\mathbf{S}}=r \hat{\mathbf{z}}, \mathbf{x}_{\mathbf{S}} \cdot \mathbf{x}^{\prime}=r r^{\prime} \cos \theta^{\prime}$, and $\left|\mathbf{x}_{S}-\mathbf{x}^{\prime}\right|=$ $\sqrt{r^{2}+r^{\prime 2}-2 r r^{\prime} \cos \theta^{\prime}}$. Hence

$X\left(\mathbf{x}_{S}\right)=-\frac{G M_{Q}^{*}}{4 \pi \delta} \int \frac{\Theta\left(R-r^{\prime}\right) r^{\prime 2} \sin \theta^{\prime} d r^{\prime} d \theta^{\prime} d \phi^{\prime}}{r^{\prime 2 \delta} \sqrt{r^{2}+r^{\prime 2}-2 r r^{\prime} \cos \theta^{\prime}}}+X_{0}$.

The integrand is independent of $\phi^{\prime}$, thus the $\phi$ integral will give a factor $2 \pi$. Now, we integrate $\theta^{\prime}$ part first. Let us define it as $I\left(r, r^{\prime}\right)$.

$$
\begin{aligned}
I\left(r, r^{\prime}\right) & =\int_{0}^{\pi}\left(r^{2}+r^{\prime 2}-2 r r^{\prime} \cos \theta^{\prime}\right)^{-1 / 2} \sin \theta^{\prime} d \theta^{\prime} \\
& =\frac{1}{r r^{\prime}}\left\{\left(r+r^{\prime}\right)-\left|r-r^{\prime}\right|\right\}
\end{aligned}
$$

Now we integrate over $r^{\prime}$.

$$
\begin{aligned}
X\left(\mathbf{x}_{S}\right) & G M_{Q}^{*} \\
= & -\frac{R}{2 \delta} \int_{0}^{R} r^{2(1-\delta)} I\left(r, r^{\prime}\right) d r^{\prime}+X_{0} \\
= & -\frac{G M_{Q}^{*}}{2 \delta}\left(\int_{0}^{r} r^{\prime 2(1-\delta)} \frac{2}{r} d r^{\prime}+\int_{r}^{R} r^{\prime 2(1-\delta)} \frac{2}{r^{\prime}} d r^{\prime}\right) \\
& +X_{0} \\
= & \frac{G M_{Q}^{*}}{2 \delta(1-\delta)}\left(\frac{r^{2(1-\delta)}}{3-2 \delta}-R^{2(1-\delta)}\right)+X_{0} .
\end{aligned}
$$

Now, one should consider $X_{0}=\frac{G M_{Q}^{*}}{2 \delta(1-\delta)} R^{2(1-\delta)}$ to get rid of the terms dependent on the boundary of near zone. One can get the following expression of the super-potential

$$
X\left(\mathbf{x}_{S}\right)=\frac{G M_{Q}^{*}}{2 \delta(1-\delta)(3-2 \delta)}\left(r_{S Q}\right)^{2(1-\delta)},
$$

where we replace $\mathbf{x}$ by $\mathbf{x}-\mathbf{y}_{Q}$ to generalize the result (Eq. (24)) and we drop the assumption $\mathbf{y}_{Q}=0$. Placing the body$\mathrm{P}$ at point $\mathrm{S}$, it can be shown that

$$
\begin{aligned}
\partial^{j} \ddot{X}_{P}= & \frac{2 G M_{Q}^{*}}{(3-2 \delta)\left(r_{P Q}\right)^{2 \delta+1}}\left[2(\delta+1)\left(\mathbf{v}_{Q} \cdot \hat{\mathbf{n}}_{P Q}\right)^{2} n_{P Q}^{j}\right. \\
& \left.-v_{Q}^{2} n_{P Q}^{j}-2\left(\mathbf{v}_{Q} \cdot \hat{\mathbf{n}}_{P Q}\right) v_{Q}^{j}+\frac{2 G M_{P}^{*}}{\left(r_{P Q}\right)^{2 \delta}} n_{P Q}^{j}\right],
\end{aligned}
$$

this expression of the derivative of the superpotential can be directly substituted in the expression for acceleration i.e., Eq. (5).

As we previously mentioned, we assume that the body-Q is much heavier than the body-P. Therefore, for mathematical simplicity, we can consider the body-Q is always stationary which implies $\mathbf{v}_{Q} \approx 0$ and $\mathbf{a}_{Q} \approx 0$.

Now these potentials [Eqs. (3), (13), and (14) are differentiated as required in Eq. (5) using Eq. (7) along with derivative of superpotential Eq. (26)] are substituted in the expression for acceleration of body-P (Eq. (5)). Acceleration of body-P $\left(a_{P}^{j}\right)$ can be obtained by substituting the expressions for the derivatives of the potentials.

$$
\begin{aligned}
a_{P}^{j}= & -\frac{G M_{Q} \epsilon^{2 \delta-1}}{r^{2 \delta+1}} n^{j}-\frac{G M_{Q} \epsilon^{2 \delta-1}}{c^{2} r^{2 \delta+1}}\left[-4\left(\mathbf{v}_{P} \cdot \hat{\mathbf{n}}\right) v_{P}^{j}\right. \\
& \left.+\left(v_{P}^{2}-\frac{2 G M_{Q} \epsilon^{2 \delta-1}}{\delta r^{2 \delta}}\right) n^{j}\right],
\end{aligned}
$$

where the mass of the body-Q $\left(M_{Q}\right)$ is related to the scale mass $M_{Q}^{*}$ by $M_{Q}^{*}=M_{Q} \epsilon^{2 \delta-1}$. In the next section, we derive the dynamical variables corresponding to the binary.

\section{The binary system and the dynamical quantities}

The EMRI under consideration comprises of a massive/ supermassive compact object (body-Q) and a stellar mass object (body-P) moving in around body- $\mathrm{Q}$. The position $\mathbf{x}$ of the body-P is written as

$\mathbf{x}=r(\cos \phi \hat{\mathbf{i}}+\sin \phi \hat{\mathbf{j}})$,

where $\phi$ is the azimuthal angle, $r$ is the distance between body-Q and body-P. Since we don't need to work with the dynamics of any body other than body-P, we drop the subscript-"P", i.e. the quantities $r_{P}, \mathbf{x}_{P}, \mathbf{v}_{P}$ and $\mathbf{a}_{P}$ will be denoted as $r, \mathbf{x}, \mathbf{v}$ and $\mathbf{a}$ and the subscript "PQ" in the quantities that are measured with reference to body- $Q$ are also dropped. i.e. $r_{P Q}, \mathbf{x}_{P Q}$ and $\hat{\mathbf{n}}_{P Q}$ are henceforth denoted as $r, \mathbf{x}, \hat{\mathbf{n}}$. In this study, we have used the following sign convention: $U(r)=+\frac{G M_{Q} \epsilon^{2 \delta-1}}{\delta r^{2 \delta}}$, and $a(r)=+\frac{d U}{d r}$. The 
differential orbit equation (for the body-P) can be written as $[22,27]$

$\frac{d^{2} u}{d \phi^{2}}+u=\frac{M_{P}^{2}}{L^{2}} \frac{d U(1 / u)}{d u}$,

where $u=1 / r, L$ is conserved angular momentum of the body-P. Therefore

$\frac{d^{2} u}{d \phi^{2}}+u=\frac{G M_{Q} M_{P}^{2}}{L^{2}}(\epsilon u)^{2 \delta-1}$

This equation has been solved for various approximations in earlier works $[37,38,40,41]$. It is very difficult to solve this differential orbit equation completely. We consider a small eccentricity approximation in which we drop the higher order terms. Let us consider a general form of the solution approximated to the first order in eccentricity-e.

$u(\phi)=\frac{1}{p}(1+e \cos m \phi)+O\left(e^{2}\right)$

Where, $e$ is eccentricity, and $m$ is a parameter which gives a precession of the orbit and $p=r\left(1+e \cos (m \phi)+O\left(e^{2}\right)\right)$. Substituting Eq. (31) into the orbit equation (30), and equating the zeroth and first order terms of eccentricity we obtain the parameter $m$ and $p$.

$$
\begin{aligned}
m & =\sqrt{2(1-\delta)} \\
p^{2(\delta-1)} & =\frac{G M_{Q} M_{P}^{2}}{L^{2}} \epsilon^{2 \delta-1}
\end{aligned}
$$

\subsection{Dynamical quantities at Newtonian order}

Henceforth, the dynamical quantities, say $\mathrm{F}$, will appear in the form of $F=F_{N}+\frac{1}{c^{2}} F_{P N}$, where $F_{N}$ denotes the Newtonian order or the 0-PN term which has been derived in this Sect. 4.1 and $F_{P N}$ denotes the 1PN correction to the quantity $\mathrm{F}$ which will be derived in the next Sect. 4.2. For example, the Newtonian order and post-Newtonian order expression for velocity of body-P are denoted as $\mathbf{v}_{N}$ and $\mathbf{v}_{P N}$ respectively while the complete $\mathrm{PN}$-corrected term is denoted as v.

The angular momentum is defined as

$$
\begin{aligned}
L & =M_{P} r^{2} \dot{\phi} \\
\therefore r^{2} \dot{\phi} & =\frac{L}{M_{P}} \\
\therefore r^{2} \dot{\phi} & =\sqrt{\frac{G M_{Q} \epsilon^{2 \delta-1}}{p^{2(\delta-1)}}}
\end{aligned}
$$

This the Newtonian order expression of the angular momentum per unit mass. As mentioned earlier (Eq. 28), the position vector is defined as $\mathbf{x}=r(\cos \phi \hat{\mathbf{i}}+\sin \phi \hat{\mathbf{j}})=r \hat{\mathbf{n}}$

Where,

$\hat{\mathbf{n}}=\cos \phi \hat{\mathbf{i}}+\sin \phi \hat{\mathbf{j}}$

$r=\frac{p}{1+e \cos m \phi}$

The velocity of the body-P is

$\mathbf{v}_{P}=\frac{d \mathbf{x}}{d t}=\frac{d \mathbf{x}}{d \phi} \frac{d \phi}{d t}=\frac{d \mathbf{x}}{d \phi} \dot{\phi}$

Using simple rules of differentiation we obtain the velocity as

$\mathbf{v}_{P}=\sqrt{\frac{G M_{Q} \epsilon^{2 \delta-1}}{p^{2 \delta}}}(e m \sin m \phi \hat{\mathbf{n}}+(1+e \cos m \phi) \hat{\boldsymbol{v}})$

4.2 Dynamical quantities with post-Newtonian correction

We use the 1PN corrected acceleration Eq. (27) to determine the 1PN correction in other dynamical variables, i.e. velocity, radial coordinate and angular frequency. In Eq. (27), we substitute the following expressions.

$$
\begin{aligned}
& \mathbf{v}_{\mathbf{P}} \cdot \hat{\mathbf{n}}=\frac{L}{p M_{P}} e m \sin m \phi \\
& v_{P}^{2}=\frac{L^{2}}{p^{2} M_{P}^{2}}(1+2 e \cos m \phi)
\end{aligned}
$$

Substituting the above expressions, and Eq. (40) into the equation of motion Eq. (27), we obtain the 1PN corrected acceleration as

$$
\begin{aligned}
\mathbf{a}= & -\frac{L^{2}}{p^{3} M_{P}^{2}}[(1+(2 \delta+1) e \cos m \phi) \hat{\mathbf{n}} \\
& -\frac{4 L^{2}}{c^{2} p^{2} M_{P}^{2}} e m \sin m \phi \hat{\boldsymbol{v}} \\
& \left.+\frac{L^{2}}{c^{2} p^{2} M_{P}^{2}}\left\{1-\frac{2}{\delta}+e \cos m \phi\left(2 \delta-\frac{2}{\delta}-5\right)\right\} \hat{\mathbf{n}}\right]
\end{aligned}
$$

which can be written as

$\mathbf{a}=\mathbf{a}_{N}+\frac{1}{c^{2}} \mathbf{a}_{P N}$

where the 1PN correction in the acceleration is

$$
\begin{aligned}
\mathbf{a}_{P N}= & \frac{L^{4}}{p^{5} M_{P}^{4}}\left[4 e m \sin m \phi \hat{\boldsymbol{v}}-\left\{1-\frac{2}{\delta}+e \cos m \phi(2 \delta\right.\right. \\
& \left.\left.\left.-\frac{2}{\delta}-5\right)\right\} \hat{\mathbf{n}}\right]
\end{aligned}
$$


From Eq. (42), we can write

$$
\frac{L^{2}}{p^{2} M_{P}^{2}}=v_{P}^{2}(1-2 e \cos m \phi)
$$

Substituting above expression into Eq. (43) we obtain

$$
\begin{aligned}
\mathbf{a}= & -\frac{L^{2}}{p^{3} M_{P}^{2}}\left[(1+(2 \delta+1) e \cos m \phi) \hat{\mathbf{n}}-4 \frac{v_{P}^{2}}{c^{2}} e m \sin m \phi \hat{\boldsymbol{v}}\right. \\
& \left.+\frac{v_{P}^{2}}{c^{2}}\left\{1-\frac{2}{\delta}+e \cos m \phi\left(2 \delta+\frac{2}{\delta}-7\right)\right\} \hat{\mathbf{n}}\right]
\end{aligned}
$$

Now, we proceed to determine the PN corrections in the other dynamical variables. Let the PN corrected angular momentum per unit mass $\left(r^{2} \dot{\phi}\right)$ and PN corrected velocity $\left(\mathbf{v}_{P}\right)$ be written as

$$
\begin{aligned}
r^{2} \dot{\phi}= & |\mathbf{r} \times \mathbf{v}|=p \sqrt{\frac{G M_{Q} \epsilon^{2 \delta-1}}{p^{2 \delta}}}\left(1+\frac{1}{c^{2}} \delta h\right), \\
\mathbf{v}_{P}= & \sqrt{\frac{G M_{Q} \epsilon^{2 \delta-1}}{p^{2 \delta}}}(e m \sin m \phi \hat{\mathbf{n}}+(1+e \cos m \phi) \hat{\boldsymbol{v}} \\
& \left.+\frac{1}{c^{2}} \delta \mathbf{v}\right),
\end{aligned}
$$

Let us evaluate the rate of change of the angular momentum per unit mass $\left(r^{2} \dot{\phi}\right)$.

$$
\begin{aligned}
& \frac{d}{d t}\left(r^{2} \dot{\phi}\right)=|\mathbf{r} \times \mathbf{a}| \\
& r|\hat{\mathbf{n}} \times \mathbf{a}| \\
& =\frac{4 G M_{Q} \epsilon^{2 \delta-1}}{c^{2} r^{2 \delta}}(\mathbf{v} . \hat{\mathbf{n}})|\hat{\mathbf{n}} \times \mathbf{v}|
\end{aligned}
$$

Where,

$$
\begin{gathered}
\mathbf{v} \cdot \hat{\mathbf{n}}=\frac{L}{p M_{P}} e m \sin m \phi \\
\hat{\mathbf{n}} \times \mathbf{v}=\frac{L}{p M_{P}}(1+e \cos m \phi) \hat{\mathbf{k}} \\
\therefore \frac{d}{d t}\left(r^{2} \dot{\phi}\right)=\frac{4 G M_{Q} \epsilon^{2 \delta-1}}{c^{2} p^{2 \delta}} \frac{L^{2}}{p^{2} M_{P}^{2}} e m \sin m \phi
\end{gathered}
$$

Where,

$$
\begin{aligned}
\frac{d}{d t}\left(r^{2} \dot{\phi}\right) & =\frac{d}{d \phi}\left(r^{2} \dot{\phi}\right) \dot{\phi} \\
& =\dot{\phi} \frac{L}{c^{2} M_{P}} \frac{d \delta h}{d \phi} \\
& =\frac{L^{2}}{c^{2} r^{2} M_{P}^{2}} \frac{d \delta h}{d \phi}
\end{aligned}
$$

Therefore,

$\frac{d \delta h}{d \phi}=\frac{4 L^{2}}{p^{2} M_{P}^{2}} e m \sin m \phi$
Evaluating the above integration, we obtain the 1PN correction to the angular momentum per unit mass $\left(r^{2} \dot{\phi}\right)$.

$$
\begin{aligned}
& \delta h=-\frac{4 L^{2} e}{p^{2} M_{P}^{2}} \cos m \phi \\
& \therefore r^{2} \dot{\phi}=\frac{L}{M_{P}}\left(1-\frac{4 L^{2} e}{c^{2} p^{2} M_{P}^{2}} \cos m \phi\right)
\end{aligned}
$$

For $\delta=\frac{1}{2}$, the above expression reduces to

$$
\begin{aligned}
r^{2} \dot{\phi} & =\frac{L}{M_{P}}\left(1-\frac{4 L^{2} e}{c^{2} p^{2} M_{P}^{2}} \cos \phi\right) \\
& =\sqrt{G M_{Q} p}\left(1-\frac{4 G M_{Q}}{c^{2} p} e \cos \phi\right)
\end{aligned}
$$

Now, we proceed to determine the 1PN correction in the velocity. Differentiating Eq. (49) with respect to time we obtain

$$
\begin{aligned}
\mathbf{a}= & \frac{d \mathbf{v}}{d t} \\
= & \dot{\phi} \frac{d \mathbf{v}}{d \phi} \\
= & \frac{L \dot{\phi}}{p M_{P}}\left[-\hat{\mathbf{n}}(1+(2 \delta-1) e \cos m \phi)+\frac{1}{c^{2}} \frac{d \delta \mathbf{v}}{d \phi}\right] \\
= & -\frac{L^{2}}{p^{3} M_{P}^{2}}[\hat{\mathbf{n}}(1+(2 \delta+1) e \cos m \phi) \\
& \left.-\frac{4 L^{2} e}{c^{2} p^{2} M_{P}^{2}} \cos m \phi \hat{\mathbf{n}}-\frac{1}{c^{2}}(1+2 e \cos m \phi) \frac{d \delta \mathbf{v}}{d \phi}\right]
\end{aligned}
$$

From Eqs. (43) and (59),

$$
\begin{aligned}
& -\frac{4 L^{2} e}{c^{2} p^{2} M_{P}^{2}} \cos m \phi \hat{\mathbf{n}}-\frac{1}{c^{2}}(1+2 e \cos m \phi) \frac{d \delta \mathbf{v}}{d \phi} \\
& =-\frac{4 L^{2} e}{c^{2} p^{2} M_{P}^{2}} m \sin m \phi \hat{\mathbf{v}}+\frac{L^{2}}{c^{2} p^{2} M_{P}^{2}}\left[1-\frac{2}{\delta}\right. \\
& \left.\quad+e \cos m \phi\left(2 \delta-\frac{2}{\delta}-5\right)\right] \hat{\mathbf{n}} \\
& \therefore \frac{d \delta \mathbf{v}}{d \phi}=\frac{L^{2}}{p^{2} M_{P}^{2}}[4 e m \sin m \phi \hat{\mathbf{v}}-e \cos m \phi(2 \delta \\
& \left.\left.\quad+\frac{2}{\delta}-3\right) \hat{\mathbf{n}}-\left(1-\frac{2}{\delta}\right) \hat{\mathbf{n}}\right] \\
& \therefore \delta \mathbf{v}=\frac{L^{2}}{p^{2} M_{P}^{2}}\left[4 e m \int \sin m \phi \hat{\mathbf{v}} d \phi-e\left(2 \delta+\frac{2}{\delta}-3\right)\right. \\
& \left.\quad \int \cos m \phi \hat{\mathbf{n}} d \phi-\left(1-\frac{2}{\delta}\right) \int \hat{\mathbf{n}} d \phi\right]
\end{aligned}
$$

Where, 


$$
\begin{aligned}
\int & \sin m \phi \hat{\mathbf{v}} d \phi \\
= & \int \sin m \phi(-\sin \phi \hat{\mathbf{i}}+\cos \phi \hat{\mathbf{j}}) d \phi \\
= & \frac{\hat{\mathbf{i}}}{2} \int\{\cos (\phi+m \phi)-\cos (\phi-m \phi)\} d \phi \\
& +\frac{\hat{\mathbf{j}}}{2} \int\{\sin (\phi+m \phi)-\sin (\phi-m \phi)\} d \phi \\
= & \frac{\hat{\mathbf{i}}}{2}\left[\frac{\sin (\phi+m \phi)}{1+m}-\frac{\sin (\phi-m \phi)}{1-m}\right] \\
& -\frac{\hat{\mathbf{j}}}{2}\left[\frac{\cos (\phi+m \phi)}{1+m}-\frac{\cos (\phi-m \phi)}{1-m}\right] \\
= & \frac{1}{1-m^{2}}[\hat{\mathbf{i}} \cos \phi \sin m \phi-\hat{\mathbf{i}} m \sin \phi \cos m \phi \\
& +\hat{\mathbf{j}} \sin \phi \sin m \phi+\hat{\mathbf{j}} m \cos \phi \cos m \phi] \\
= & \frac{1}{2 \delta-1}[\sin m \phi \hat{\mathbf{n}}+m \cos m \phi \hat{\mathbf{v}}]
\end{aligned}
$$

Similarly, we determine

$$
\int \cos m \phi \hat{\mathbf{n}} d \phi=-\frac{1}{2 \delta-1}[\cos m \phi \hat{\boldsymbol{v}}+m \sin m \phi \hat{\mathbf{n}}]
$$

which is valid for the case $\delta \neq \frac{1}{2}$.

$$
\begin{aligned}
\therefore \delta \mathbf{v}= & \frac{L^{2}}{p^{2} M_{P}^{2}}\left[\frac { e } { 2 \delta - 1 } \left\{m \sin m \phi\left(2 \delta+\frac{2}{\delta}+1\right) \hat{\mathbf{n}}\right.\right. \\
& \left.\left.-\cos m \phi\left(6 \delta-\frac{2}{\delta}-5\right) \hat{\boldsymbol{v}}\right\}+\left(1-\frac{2}{\delta}\right) \hat{\boldsymbol{v}}\right]
\end{aligned}
$$

The 1PN corrected velocity of the body-P is given below.

$$
\begin{aligned}
\mathbf{v}= & \frac{L}{p M_{P}}[e m \sin m \phi \hat{\mathbf{n}}+(1+e \cos m \phi) \hat{\boldsymbol{v}} \\
& +\frac{L^{2}}{c^{2} p^{2} M_{P}^{2}}\left[\frac { e } { 2 \delta - 1 } \left\{m \sin m \phi\left(2 \delta+\frac{2}{\delta}+1\right) \hat{\mathbf{n}}\right.\right. \\
& \left.\left.\left.-\cos m \phi\left(6 \delta-\frac{2}{\delta}-5\right) \hat{\boldsymbol{v}}\right\}+\left(1-\frac{2}{\delta}\right) \hat{\boldsymbol{v}}\right]\right]
\end{aligned}
$$

So we have obtained two dynamical quantities, namely acceleration-a and velocity-v (Eqs. 47 and 66 respectively). We would like to mention that, in the next section (Sect. 5) we shall discuss the 1PN corrected average energy radiation rate from an EMRI. We will use the mass quadrupole tensor which requires us to derive the 1-PN corrected expressions for $r$ and $\dot{\phi}$. It is very much difficult to analytically derive the 1PN corrected $r$ and $\dot{\phi}$ using Eqs. (43), (57), and (66) since we are considering the case of non-circular orbit (i.e., $e \neq 0$ ). For mathematical simplicity, we restrict our calculations to the circular orbit in which eccentricity $e=0$ and we discuss the dynamical quantities in the next Sect. 4.3.
4.3 $1 \mathrm{PN}$ corrected dynamical quantities for circular orbit-case

Substituting $e=0$ in expression for the acceleration (Eq. 43 ) reduces it to,

$\mathbf{a}=-\frac{G M_{Q} \epsilon^{2 \delta-1}}{p^{2 \delta+1}} \hat{\mathbf{n}}+\frac{1}{c^{2}} \frac{G^{2} M_{Q}^{2} \epsilon^{2(2 \delta-1)}}{\delta p^{4 \delta+1}}(2-\delta) \hat{\mathbf{n}}$,

The expression for velocity at the Newtonian order (OPN) can be written as $v_{N}^{2}=\frac{G M_{Q}}{p^{2 \delta}} \epsilon^{2 \delta-1}$ as derived in Eq. (40). After substituting this expression, the post-Newtonian parameter $\left(v_{N}^{2} / c^{2}\right)$ becomes evidently apparent. Hence we can simplify Eq. (67),

$\mathbf{a}=-\frac{G M_{Q} \epsilon^{2 \delta-1}}{p^{2 \delta+1}}\left[1-\frac{v_{N}^{2}}{c^{2}} \frac{(2-\delta)}{\delta}\right] \hat{\mathbf{n}}$.

In Kepler-Newton (KN) case, $\delta=1 / 2$, and the acceleration $\left(\mathbf{a}_{K N}\right)$ reduces to $([42,43])$

$\mathbf{a}_{K N}=-\frac{G M_{Q}}{p^{2}}\left[1-3 \frac{v_{K N}^{2}}{c^{2}}\right] \hat{\mathbf{n}}$,

where $v_{K N}^{2}=\frac{G M_{Q}}{p}$.

Post-Newtonian corrections are only valid as long as $\left(\frac{v_{N}}{c}\right)^{2} \ll 1$ and the PN correction is much smaller than the Newtonian order part. This can be simplified to give the following condition

$1 \gg\left(\frac{v_{N}}{c}\right)^{2}\left(\frac{2-\delta}{\delta}\right)=\frac{G M_{Q} \epsilon^{(2 \delta-1)}}{p^{2 \delta} c^{2}} \frac{2-\delta}{\delta} \Longrightarrow \delta>0$.

We shall consider the cases where $\delta>0$.

Now, we proceed to determine the PN corrections in the other dynamical variables. The PN corrected angular momentum per unit mass $\left(r^{2} \dot{\phi}\right)$ and PN corrected velocity $\left(\mathbf{v}_{P}\right)$ become

$r^{2} \dot{\phi}=p \sqrt{\frac{G M_{Q} \epsilon^{2 \delta-1}}{p^{2 \delta}}}$

Since circular orbits are considered, the radial component of the velocity is zero. Hence the velocity can be written as $v=r \dot{\phi}$. Also the relation between acceleration $\mathbf{a}$ and the velocity $\mathbf{v}$ can be given as:

$$
\begin{aligned}
\mathbf{a} & =\frac{d \mathbf{v}}{d t}=\frac{d \mathbf{v}}{d \phi} \frac{d \phi}{d t}=\frac{d \mathbf{v}}{d \phi} \dot{\phi} \\
\frac{d \delta \mathbf{v}}{d \phi} & =\frac{1}{c^{2}} \frac{(2-\delta)}{\delta}\left[\frac{G M_{Q} \epsilon^{2 \delta-1}}{p^{2 \delta}}\right]^{3 / 2} \hat{\mathbf{n}},
\end{aligned}
$$


$\therefore \delta \mathbf{v}=-\frac{1}{c^{2}} \frac{(2-\delta)}{\delta}\left[\frac{G M_{Q} \epsilon^{2 \delta-1}}{p^{2 \delta}}\right]^{3 / 2} \hat{\mathbf{v}}$.

Hence, the velocity $\mathbf{v}_{P}$ of the body-P corrected up to the first order Post-Newtonian term is

$\mathbf{v}_{P}=\sqrt{\frac{G M_{Q} \epsilon^{2 \delta-1}}{p^{2 \delta}}}\left[1-\frac{1}{c^{2}} \frac{G M_{Q} \epsilon^{2 \delta-1}}{p^{2 \delta}} \frac{(2-\delta)}{\delta}\right] \hat{\boldsymbol{v}}$.

This above expression can also be obtained by putting $e=0$ in Eq. (66). Further this expression can be used to derive the expression for radial coordinate $(r)$ and the angular velocity $(\dot{\phi})$.

Substituting Eq. (75) in the following relation

$\mathbf{v}_{P}=\frac{d \mathbf{x}}{d t}=\frac{d \mathbf{x}}{d \phi} \frac{d \phi}{d t}=r \dot{\phi} \hat{\boldsymbol{v}}=\frac{r^{2} \dot{\phi}}{r} \hat{\boldsymbol{v}}$,

and using $r^{2} \dot{\phi}$ from Eq. (71), we obtain

$$
\begin{aligned}
& \sqrt{\frac{G M_{Q} \epsilon^{2 \delta-1}}{p^{2 \delta}}}\left[1-\frac{1}{c^{2}} \frac{G M_{Q} \epsilon^{2 \delta-1}}{p^{2 \delta}} \frac{(2-\delta)}{\delta}\right] \hat{\boldsymbol{v}} \\
& =\frac{r^{2} \dot{\phi}}{r} \hat{\boldsymbol{v}}=\frac{p}{r} \sqrt{\frac{G M_{Q} \epsilon^{2 \delta-1}}{p^{2 \delta}}} \hat{\boldsymbol{v}},
\end{aligned}
$$

which implies

$\frac{p}{r}=\left[1-\frac{1}{c^{2}} \frac{G M_{Q} \epsilon^{2 \delta-1}}{p^{2 \delta}} \frac{(2-\delta)}{\delta}\right]$.

Therefore, the radial coordinate $(r)$ corrected up to the first order Post-Newtonian term is

$r=p\left[1+\frac{1}{c^{2}} \frac{G M_{Q} \epsilon^{2 \delta-1}}{p^{2 \delta}} \frac{(2-\delta)}{\delta}\right]+O\left(c^{-4}\right)$.

Next, we derive the expression for the angular velocity $\dot{\phi}$. Substituting Eq. (79) into Eq. (71) and simplifying for $\dot{\phi}$, we obtain

$\dot{\phi}=\frac{1}{p} \sqrt{\frac{G M_{Q} \epsilon^{2 \delta-1}}{p^{2 \delta}}}\left[1-\frac{1}{c^{2}} \frac{2 G M_{Q} \epsilon^{2 \delta-1}}{\delta p^{2 \delta}}(2-\delta)\right]$.

In the next section, we discuss the average energy radiation rate of EMRI using the expressions of PN corrected quantities derived here.

\section{Average energy radiation rate from an EMRI}

Power law potential with $\delta>0$, would ensure that the potential dies out at large distance from the source mass. It should be noted that the metric which leads to power law potential tends to become Minkowskian at large distance from the mass distribution. Assuming the quadrupole formula applies for power law potential, the average energy radiation rate $\left\langle\frac{d E}{d t}\right\rangle$ from a binary can be given by the following expression $[36,42,43]$.

$\left\langle\frac{d E}{d t}\right\rangle=-\frac{G}{45 c^{5}} \frac{1}{T} \int_{0}^{T}\left(\dddot{D}^{\alpha \beta}\right)^{2} d t$

where the mass quadrupole tensor $D^{\alpha \beta}$ is defined as [42]

$D^{\alpha \beta}=M_{P}\left(3 x^{\alpha} x^{\beta}-|\mathbf{x}|^{2} \delta^{\alpha \beta}\right)$.

The effects like the dephasing of the orbital motion due dynamical friction in the presence of matter distribution are not taken into consideration at this stage because we assume small deviations of $\delta$ from $\delta_{K N}=1 / 2$ i.e., small deviation from $\mathrm{KN}$ potential. This also means that the mass distribution away from body-Q and near body-P is negligible.

Now the quadrupole tensor $D^{\alpha \beta}$ can be simplified as follows,

$$
\begin{aligned}
\dddot{D}^{\alpha \beta}= & \frac{d^{3}}{d t^{3}}\left[M_{P}\left(3 x^{\alpha} x^{\beta}-|\mathbf{x}|^{2} \delta^{\alpha \beta}\right)\right] \\
= & M_{P} \frac{d^{2}}{d t^{2}}\left[3 \dot{x}^{\alpha} x^{\beta}+3 x^{\alpha} \dot{x}^{\beta}-2 \mathbf{x} \cdot \dot{\mathbf{x}} \delta^{\alpha \beta}\right] \\
= & M_{P} \frac{d}{d t}\left[3 \ddot{x}^{\alpha} x^{\beta}+6 \dot{x}^{\alpha} \dot{x}^{\beta}+3 x^{\alpha} \ddot{x}^{\beta}\right. \\
& \left.-2 \dot{x}^{2} \delta^{\alpha \beta}-2 \mathbf{x} \cdot \ddot{\mathbf{x}} \delta^{\alpha \beta}\right] \\
= & M_{P}\left[3 \dddot{x}^{\alpha} x^{\beta}+9 \ddot{x}^{\alpha} \dot{x}^{\beta}+9 \dot{x}^{\alpha} \ddot{x}^{\beta}+3 x^{\alpha} \dddot{x}^{\beta}\right. \\
& \left.-6 \dot{\mathbf{x}} \cdot \ddot{\mathbf{x}} \delta^{\alpha \beta}-2 \mathbf{x} . \dddot{\mathbf{x}} \delta^{\alpha \beta}\right]
\end{aligned}
$$

Hence, $\left(\dddot{D}^{\alpha \beta}\right)^{2}$ can be written as

$$
\begin{aligned}
\left(\dddot{D}^{\alpha \beta}\right)^{2}= & \left(\dddot{D}^{\alpha \beta}\right)\left(\dddot{D}_{\alpha \beta}\right) \\
= & 6 M_{P}^{2}\left[3 \dddot{x}^{2} x^{2}+18(\dddot{\mathbf{x}} \cdot \ddot{\mathbf{x}})(\dot{\mathbf{x}} \cdot \mathbf{x})+18(\dddot{\mathbf{x}} \cdot \dot{\mathbf{x}})(\ddot{\mathbf{x}} \cdot \mathbf{x})\right. \\
& \left.+(\mathbf{x} \cdot \dddot{\mathbf{x}})^{2}+27 \dot{\mathbf{x}}^{2} \ddot{\mathbf{x}}^{2}+9(\dot{\mathbf{x}} \cdot \ddot{\mathbf{x}})^{2}-12(\dddot{\mathbf{x}} \cdot \mathbf{x})(\ddot{\mathbf{x}} \cdot \dot{\mathbf{x}})\right]
\end{aligned}
$$

where the acceleration terms $(\ddot{\mathbf{x}})$ can be written as the gradient of the potential or the magnitude of the force corresponding to this acceleration is given as $\mathbf{F}_{\mathbf{a}}=M_{P}|\mathbf{a}|=\frac{\partial V}{\partial r}$, where $V$ is the potential energy. Therefore, the acceleration of the body-P becomes

$\mathbf{a}=\frac{1}{M_{P}} \frac{\partial V}{\partial r} \hat{\mathbf{n}}+\frac{1}{c^{2}} \mathbf{a}_{P N}$

Substituting the above expression of a into Eq. (84) and after some simplification, we obtain the following expression for $\left(\dddot{D}^{\alpha \beta}\right)^{2}([42,44])$ :

$\left(\dddot{D}^{\alpha \beta}\right)^{2}=24\left[\left(r \dot{r} \frac{\partial^{2} V}{\partial r^{2}}+3 \dot{r} \frac{\partial V}{\partial r}\right)^{2}+12 r^{2} \dot{\phi}^{2}\left(\frac{\partial V}{\partial r}\right)^{2}\right]$ 


$$
\begin{aligned}
& +\frac{24 M_{P}}{c^{2}}\left[2 r\left(\dot{r} \frac{\partial^{2} V}{\partial r^{2}} \mathbf{x}+3 \frac{\partial V}{\partial r} \dot{\mathbf{x}}\right) \cdot \dot{\mathbf{a}}_{P N}\right. \\
& +\left\{9 \frac{\partial V}{\partial r}\left(\dot{r}^{2}+2 r^{2} \dot{\phi}^{2}\right) \hat{\mathbf{n}}+3 \dot{r} \frac{\partial^{2} V}{\partial r^{2}}(3 \dot{r} \mathbf{x}\right. \\
& \left.\left.-r \dot{\mathbf{x}})+9 \dot{r} \frac{\partial V}{\partial r} \dot{\mathbf{x}}\right\} . \mathbf{a}_{P N}\right],
\end{aligned}
$$

For circular orbits $\dot{r}=0$, therefore Eq. (86) reduces to the following expression.

$$
\begin{aligned}
\left(\dddot{D}^{\alpha \beta}\right)^{2}= & 24\left[12\left(\frac{\partial V}{\partial r}\right)^{2} r^{2} \dot{\phi}^{2}+\frac{M_{P}}{c^{2}}\right. \\
& \left.\left\{18^{2} \dot{\phi}^{2} \frac{\partial V}{\partial r} \hat{\mathbf{n}} \cdot \mathbf{a}_{P N}+6 r \frac{\partial V}{\partial r} \mathbf{v}_{P} \cdot \dot{\mathbf{a}}_{P N}\right\}\right]
\end{aligned}
$$

Here, the time derivative of the PN correction term $\dot{\mathbf{a}}_{P N}$ of the acceleration is

$\dot{\mathbf{a}}_{P N}=\frac{d \mathbf{a}_{P N}}{d t}=\frac{d \mathbf{a}_{P N}}{d \phi} \frac{d \phi}{d t}=\frac{d \mathbf{a}_{P N}}{d \phi} \dot{\phi}$.

Therefore,

$\dot{\mathbf{a}}_{P N}=\left[\frac{G M_{Q} \epsilon^{2 \delta-1}}{p^{2 \delta}}\right]^{5 / 2} \frac{(2-\delta)}{\delta p^{2}} \hat{\mathbf{v}}+O\left(c^{-2}\right)$,

where the higher order terms are ignored as they will contribute to the $2 \mathrm{PN}$ or higher orders, which is out of the scope of this current study. We can also write,

$$
\begin{aligned}
V(r) & =\frac{G M_{Q} M_{P}}{2 \delta r^{2 \delta} \epsilon^{2 \delta-1}}, \\
\mathbf{v}_{P} \cdot \dot{\mathbf{a}}_{P N} & =\frac{G^{3} M_{Q}^{3} \epsilon^{3(2 \delta-1)}}{\delta p^{2(3 \delta+1)}}(2-\delta)+O\left(c^{-2}\right), \\
\hat{\mathbf{n}}_{. \mathbf{a}_{P N}}= & \frac{G^{2} M_{Q}^{2} \epsilon^{2(2 \delta-1)}}{\delta p^{4 \delta+1}}(2-\delta)+O\left(c^{-2}\right) .
\end{aligned}
$$

Now substituting Eqs. (79), (80), and the above expressions into Eq. (87), we obtain

$$
\begin{aligned}
\left(\dddot{D}^{\alpha \beta}\right)^{2}= & \frac{288 G^{3} M_{Q}^{3} M_{P}^{2} \epsilon^{3(2 \delta-1)}}{p^{2(3 \delta+1)}}\left[1-\frac{1}{c^{2}} \frac{2 G M_{Q} \epsilon^{2 \delta-1}}{\delta p^{2 \delta}}(2\right. \\
& -\delta)(3+2 \delta)] .
\end{aligned}
$$

The average rate of the energy radiation mentioned in Eq. (81) can be written as,

$\left\langle\frac{d E}{d t}\right\rangle=-\frac{G}{45 c^{5}} \frac{1}{2 \pi} \int_{0}^{2 \pi}\left(\dddot{D}^{\alpha \beta}\right)^{2} d \phi$,

where $\dot{\phi}=2 \pi / T$. In the case of circular orbits, the integrand is independent of the variable $\phi$. Therefore, the above expression of the average energy radiation rate reduces to

$\left\langle\frac{d E}{d t}\right\rangle=-\frac{G}{45 c^{5}}\left(\dddot{D}^{\alpha \beta}\right)^{2}$.
Thus

$$
\begin{aligned}
\left\langle\frac{d E}{d t}\right\rangle= & -\frac{32 G^{4} M_{Q}^{3} M_{P}^{2} \epsilon^{3(2 \delta-1)}}{5 c^{5} p^{2(3 \delta+1)}}\left[1-\frac{1}{c^{2}} \frac{2 G M_{Q} \epsilon^{2 \delta-1}}{\delta p^{2 \delta}}(2\right. \\
& -\delta)(3+2 \delta)] .
\end{aligned}
$$

The condition for using PN approximations to evaluate average energy radiation rate for power law potential is

$1 \gg \frac{G M_{Q}}{c^{2} p^{2 \delta}} \frac{(2-\delta)(3+2 \delta)}{\delta}$.

For a given EMRI and in a range of radial distance, this condition can be narrowed down to a cut-off $\delta_{0}$ below which PN approximations cannot be used to work out the average energy radiation rate. This cut-off depends on the properties of the binary under consideration.

Equation (96) shows the energy radiation from the binaries following power law potential with the assumptions that (i) the scale length $(\epsilon)$ is very small compared to the distance between two bodies i.e. $r_{P Q}$, (ii) the body-Q is much heavier than the body-P, therefore the motion of the body-Q is negligible, and it can be treated as a stationary body and (iii) the body-P is moving in the circular orbit around $\mathrm{Q}$.

\section{Comparison of power law potential with the Kepler-Newton potential}

Consider an example of an EMRI system of a massive black hole, and a star as massive as the sun.

Let the properties of the massive compact object be: mass, $M_{Q}=10^{34} \mathrm{~kg}$, the scale radius, $\epsilon=10^{7} \mathrm{~m}$ and the properties of the star or the stellar mass object be: mass, $M_{P}=10^{30} \mathrm{~kg}$.

In this section, we compare the velocity curves, the average energy radiation rate and the orbital frequency of the binary in the PL potential and in the KN Potential. The PN corrections are much smaller than the Newtonian values of the evaluated quantities. Hence, in the magnitude scales considered below, the graphs of the Newtonian and the PN corrected quantities will apparently overlap.

\subsection{Velocity profile}

The comparison of the velocity curves for power law potential (Eq. (75)) and Kepler-Newton potential is shown in this subsection. For $0<\delta<0.5$, the velocity falls more slowly in the case of power law (PL) potential as compared to that in KN potential case which is usually observed in astrophysical scenarios (Fig. 1a). For the case of $0.5<\delta$, the velocity for PL potential drops faster (compared to that in the case of $\mathrm{KN}$ potential i.e., vacuum environment) with increasing 
radial distance (Fig. 1b). Therefore, $0.5<\delta$ is astrophysically improbable.

\subsection{Average energy radiation rate}

Next, we compare the average energy radiation rate from an EMRI in PL potential and that in KN Potential.

Average energy radiation rate from a system in $\mathrm{KN}$ potential can be determined by substituting $\delta=1 / 2$ in our final result (Eq. (96))

$$
\left\langle\frac{d E}{d t}\right\rangle_{K N P}=-\frac{32 G^{4} M_{Q}^{3} M_{P}^{2}}{5 c^{5} p^{5}}\left(1-\frac{1}{c^{2}} \frac{24 G M_{Q}}{p}\right) .
$$

The comparisons of the two energy radiation rates, Eq. (98), and Eq. (96) with $\delta \neq 0.5$, are shown in the Fig. 2 for different $\delta$-s.

In the EMRI considered above, we focus on the region that satisfies the condition for weak field zone (Eq. 97) where the radial distance $p \approx 10^{10}$ meter and the corresponding $\delta_{0}$ is close to 0.5 . For $\delta=0.45<0.5$, the energy radiation rate is greater than that from $\mathrm{KN}$ potential (Fig. 2a). For $\delta>0.5$, the energy radiation rate is smaller than that from $\mathrm{KN}$ potential (Fig. 2b). However, as we mentioned before, $\delta>0.5$ is astrophysically improbable.

\subsection{Orbital frequency}

Presence of matter distribution around a binary affects the dynamics of the binary. The effect of dynamical friction as studied in [45-47] is ignored here. Let's consider a power law (where the power is $\delta$ ) such that it has a small deviation $(\Delta$ ) in the power from the Kepler-Newton potential $\left(\delta_{K N}=1 / 2\right)$ which could be because of the presence of matter distribution around the EMRI. Accordingly, from the Eq. (80), the orbital frequency $\dot{\phi}$ can be written as

$\dot{\phi}=\frac{1}{p} \sqrt{\frac{G M_{Q} \epsilon^{1-2 \Delta}}{p^{(1-2 \Delta)}}}\left[1-\frac{1}{c^{2}} \frac{2 G M_{Q} \epsilon^{-2 \Delta}}{(1 / 2-\Delta) p^{(1-2 \Delta)}}(3 / 2+\Delta)\right]$.

When $\Delta>0$ as shown for $\delta=0.45$, which is also the case where we expect non-vacuum environment, the orbital frequency is increased (Fig. 3a). While in the case where $\Delta<$ 0 , eg. $\Delta=-0.05$, i.e., $\delta=0.55$ we get orbital frequency lower than that of an EMRI in KN potential but this case astrophysically improbable.

From the observation data of the orbital frequency of EMRI, the value of $\Delta$ can be obtained which gives the precise power law potential which can be used to get the matter density profile around the central massive compact object. It can also be noted from Fig. 3a that for the case where $\delta<0.5$, the orbital frequency $\left(\approx 10^{-4}\right)$ is closer to the LISA frequency range. Which means that presence of the mass distribution leads to possibility of observing the inspiral using LISA in the lower frequency region; while LISA would not be able to detect the EMRI comprised of only the two point objects.

\section{Conclusion}

In this paper, we consider an extreme mass ratio inspiral (EMRI) in a system where the massive/supermassive object lies in a non vacuum region such that the effective weak field gravitational potential can have a more general power law potential. The main motivation to consider power law potential is to get nearly flat profile of orbital velocity which is observed in astrophysical scenarios (e.g. galactic rotation curve). Using the Newtonian gravitational potential $(U)$ and the PN potentials $\left(U^{j}, \Psi\right.$ and $\left.X\right)$, we derive the acceleration of a body in the PL potential. Considering a small eccentricity we derive the PN corrected acceleration and velocity. Analytical calculation of the PN corrections to the quantities like orbital frequency and the average energy radiation rate is very difficult and out of scope of the current paper. We consider circular orbits for the ease of calculations. Using this, we derive other PN corrected dynamical variables. Subsequently, we obtain the mass quadrupole tensor which is ultimately used to obtain the average energy radiation rate.

Next, we discuss an example of an EMRI. We calculate the average energy radiation rate from this EMRI in power law potential at different $\delta$-s and compared them with that from the same EMRI at $\delta=0.5$ i.e. the $\mathrm{KN}$ potential case. When $\delta$ is less than a particular cut-off $\delta_{0}$, we cannot use PN approximations because it violates the conditions that the value of 1-PN part should be much less than 0-PN part. The important results we get here are,

- The average energy radiation rate that we have derived is applicable for every EMRI which is surrounded by a matter distribution that can give power law potential in the region around the massive/supermassive compact object. We show that the average energy radiation rate is higher in general PL potential case as compared to that in $\mathrm{KN}$ potential.

- We use the comparison of orbital frequency of an EMRI in PL and KN potentials to show how matter distribution (e.g. dark matter distribution) causes change in the signals. This can be used to further investigate the quantities like frequencies of the gravitational waves radiated from such an EMRI. We have also shown how the presence of matter distribution leads to higher frequency which may push the binary in the detectable range of future detectors like LISA. The deviation of the observed orbital frequen- 


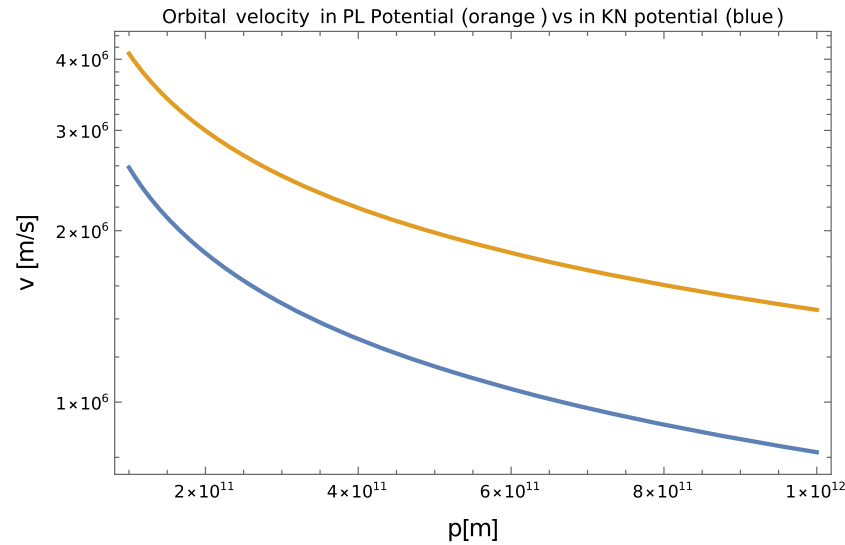

(a) For $\delta=0.45$ (i.e. $\delta<0.5)$

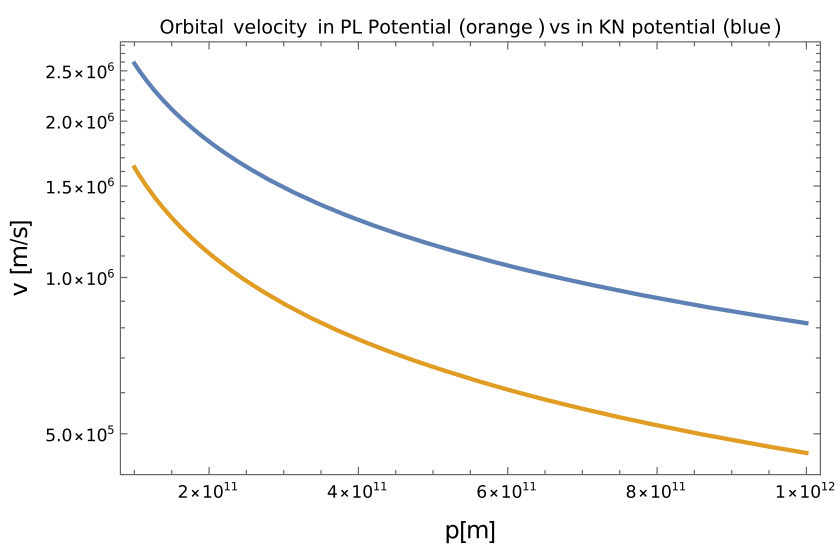

(b) For $\delta=0.55$ (i.e. $\delta>0.5)$

Fig. 1 Figure shows velocity of the smaller object in the extreme mass ratio binary in power law (PL) potential (orange curve) and in Kepler-Newton $(\mathrm{KN})$ potential (blue curve) with respect to radial distance between the two bodies

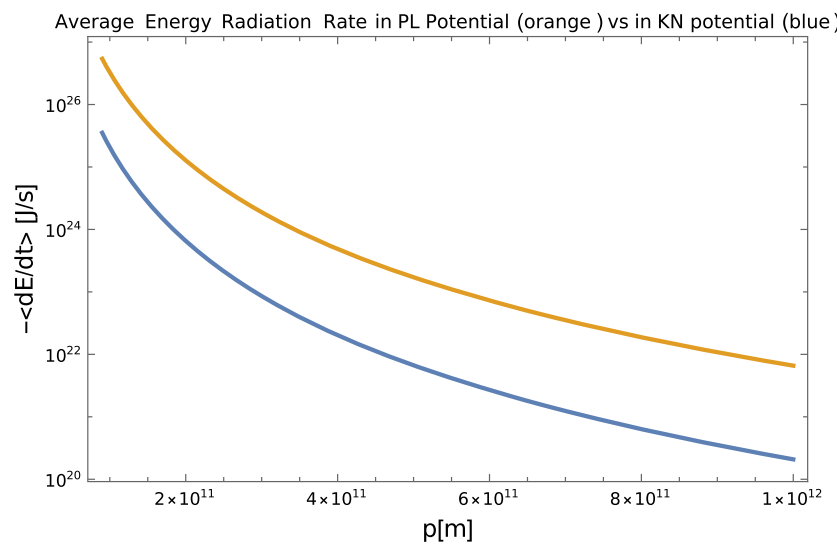

(a) $\delta=0.45($ i.e. $\delta<0.5)$

Fig. 2 Figure shows variation of 1-PN corrected rate of energy radiation from an extreme mass ratio binary in power law (PL) potential (orange curve) and in Kepler-Newton (KN) potential (blue curve) with

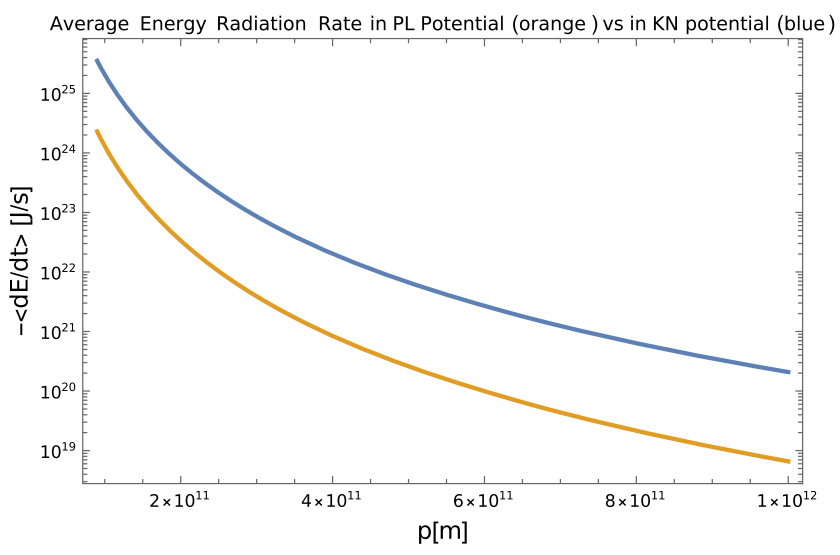

(b) $\delta=0.55$ (i.e. $\delta>0.5)$

respect to radial distance $(p)$ between the two bodies. Note that the $y$ axis denotes the negative of average of rate of change of energy. Which means that the system is losing energy

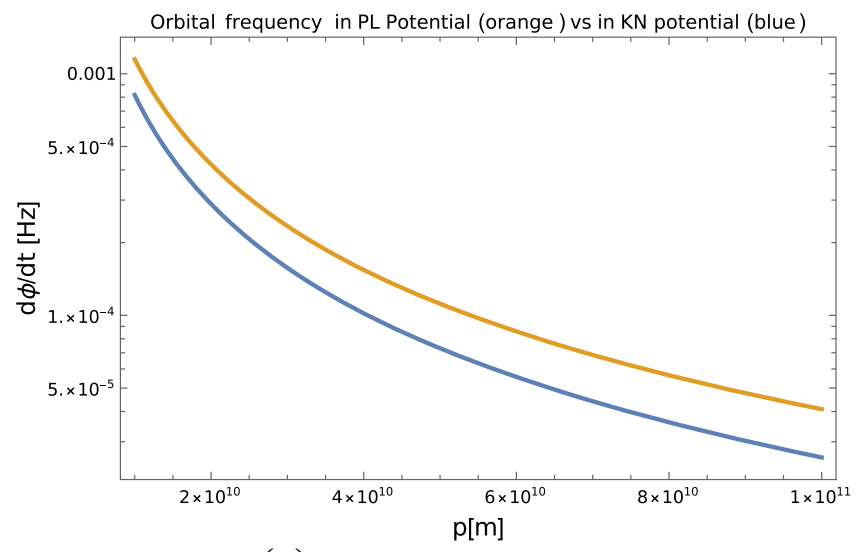

(a) $\delta=0.45($ i.e. $\delta<0.5)$,

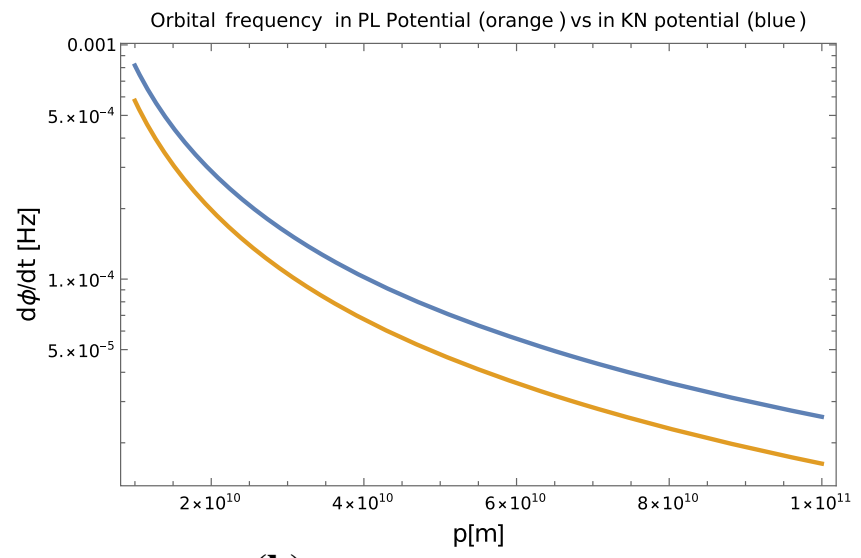

(b) $\delta=0.55($ i.e. $\delta>0.5)$

Fig. 3 Figure shows the comparison of the orbital frequency of the extreme mass ratio binary in power law (PL) potential (orange curve) and in Kepler-Newton $(\mathrm{KN})$ potential (blue curve) with respect to radial distance $(p)$ between the two bodies 
cies from the frequency of a similar binary in KN potential can be used to evaluate the value of $\delta$ and that can be used to find out the effective potential and subsequently, can calculate the mass density profile around the binary.

We have compared the leading order (Newtonian order) expressions for the dynamical variables and the average energy radiation rate as derived in Sects. 4.2 and 5 since the higher order terms are extremely small. However, the derivation of the PN contributions as provided in this paper, opens up an opportunity to model and study the dynamics of such a binary system as well as the gravitational waveforms originating from them. Further scopes could include (1) obtaining the strains of gravitational waves from such a system, (2) derivation and incorporation of higher PN orders and (3) use the waveforms and check their detectability using upcoming detectors like DECIGO and LISA. These developments can be expected from our upcoming works.

Acknowledgements C.N.G. would like to acknowledge the support of the International Center for Cosmology, CHARUSAT, India for funding the work.

Data Availability Statement This manuscript has no associated data or the data will not be deposited. [Authors' comment: This is a totally theoretical work. Therefore, no data is used here.]

Open Access This article is licensed under a Creative Commons Attribution 4.0 International License, which permits use, sharing, adaptation, distribution and reproduction in any medium or format, as long as you give appropriate credit to the original author(s) and the source, provide a link to the Creative Commons licence, and indicate if changes were made. The images or other third party material in this article are included in the article's Creative Commons licence, unless indicated otherwise in a credit line to the material. If material is not included in the article's Creative Commons licence and your intended use is not permitted by statutory regulation or exceeds the permitted use, you will need to obtain permission directly from the copyright holder. To view a copy of this licence, visit http://creativecomm ons.org/licenses/by/4.0/.

Funded by SCOAP 3 .

\section{References}

1. Y. Sofue, V. Rubin, Ann. Rev. Astron. Astrophys. 39(1), 137 (2001). https://doi.org/10.1146/annurev.astro.39.1.137

2. M. Milgrom, Astrophys. J. 270, 365-370 (1983)

3. E.A., Evstigneeva, (Y.A. Nikolaev publisher, 2004), p. 180-196

4. D.C. Rodrigues, P.S. Letelier, I.L. Shapiro, J. Cosmol. Astropart. Phys. 2010(04), 020 (2010)

5. B.E. Meierovich, Phys. Rev. D 87, 103510 (2013). https://doi.org/ 10.1103/PhysRevD.87.103510

6. F. Zwicky, Gen. Relativ. Gravit. 41(1), 207-224 (2009)

7. V.C. Rubin, W.K. Ford, N. Thonnard, Astrophys. J. 238, 471-487 (1980)

8. V.C. Rubin, W.K. Ford, Astrophys. J. 159, 379 (1970)

9. E. Díaz, A. Zandivarez, M.E. Merchán et al., Astrophys. J. 629, 158 (2005). https://doi.org/10.1086/431234
10. F. Donato, G. Gentile, P. Salucci, Mon. Not. R. Astron. Soc. 353, L17 (2004). https://doi.org/10.1111/j.1365-2966.2004.08220.x

11. J.F. Navarro, C.S. Frenk, S.D.M. White, Astrophys. J. 462, 563 (1996)

12. U. Banik, D. Dey, K. Bhattacharya et al., Gen. Relativ. Gravit. 49, 116 (2017). https://doi.org/10.1007/s10714-017-2284-x

13. D. Dey, K. Bhattacharya, T. Sarkar, Gen. Relativ. Gravit. 47, 103 (2015)

14. D. Dey, K. Bhattacharya, T. Sarkar, Phys. Rev. D 87(10), 103505 (2013)

15. D. Dey, K. Bhattacharya, T. Sarkar, Phys. Rev. D 88(8), 083532 (2013)

16. L.S. The, S.D.M. White, Astron. J. 95, 1642 (1988)

17. J.D. Bekenstein, Phys. Rev. D 70(8), 083509 (2004)

18. J.W. Moffat, J. Cosmol. Astropart. Phys. 2005, 5 (2005). https:// doi.org/10.1088/1475-7516/2005/05/003

19. J.W. Moffat, J. Cosmol. Astropart. Phys. 2006(03), 004 (2006)

20. J.R. Brownstein, J.W. Moffat, Astrophys. J. 636, 2 (2006). https:// doi.org/10.1086/498208

21. H.A. Munera, C. Delgado-Correal, Res. Notes AAS 4(9), 151 (2020)

22. H. Goldstein, Addison-Wesley (1950)

23. J.M.A. Danby, Willmann-Bell, 2nd ed., rev. \& enl (1988)

24. S.R. Valluri, P.A. Wiegert, J. Drozd, M. Da Silva, Mon. Not. R. Astron. Soc. 427(3), 2392 (2012)

25. D. Lynden-Bell, S. Jin, Mon. Not. R. Astron. Soc. 386(1), 245 (2008). https://doi.org/10.1111/j.1365-2966.2008.13018.x

26. S.R. Valluri, P. Yu, G.E. Smith, P.A. Wiegert, Mon. Not. R. Astron. Soc. 358(4), 1273 (2005)

27. L. Landau, E. Lifshitz, 3 edition (Butterworth-Heinemann, 1976)

28. P.C. Peters, J. Mathews, Phys. Rev. 131, 435 (1963)

29. B. Leor, C. Curt, Phys. Rev. D 69(8), 1550-2368 (2004). https:// doi.org/10.1103/PhysRevD.69.082005

30. P. Amaro-Seoane, S. Aoudia, S. Babak et al., Class. Quantum Gravity 29, 124016 (2012). https://doi.org/10.1088/0264-9381/29/12/ 124016

31. G. Kostas, Class. Quantum Gravity 22(15), S605-S659 (2005). https://doi.org/10.1088/0264-9381/22/15/004

32. J.R. Gair, M. Vallisneri, S.L. Larson et al., Living Rev. Relativ. 16(1), 1433-8351 (2013). https://doi.org/10.12942/lrr-2013-7

33. J.R. Gair et al., (2017) https://doi.org/10.1088/1742-6596/840/1/ 012021

34. R. Fujita, M. Shibata, Phys. Rev. D 102, 064005 (2020). https:// doi.org/10.1103/PhysRevD.102.064005

35. M.L. Katz, A.J.K. Chua, L. Speri et al., (2021) arXiv:2104.04582

36. E. Poisson, And Will (Cambridge Press, C. M., 2014)

37. C. Struck, Mon. Not. R. Astron. Soc. 446(3), 3139 (2015)

38. C. Struck, Astron. J. 131(3), 1347 (2006)

39. J. Binney, S. Tremaine, (Princeton University Press, Princeton, 1987)

40. P. Bambhaniya, A.B. Joshi, D. Dey, P.S. Joshi, Phys. Rev. D 100(12), 124020 (2019). https://doi.org/10.1103/PhysRevD.100. 124020

41. S.R. Valluri, C. Wilson, W. Harper, J. Hist. Astron. 28, 13 (1997) https://doi.org/10.1177/002182869702800102

42. D.D. Dionysiou, Astrophysi. Space Sci. 125(1), 115-125 (1986)

43. R.V. Wagoner, C.M. Will, Astrophys. J. 210, 764-775 (1976)

44. H. Dehnen, F. Ghaboussi, Nuovo Cimento B, Serie 11 (ISSN 03693554), vol. 89 B, p. 131-138 (1985)

45. J.D. Bekenstein, R. Zamir, Astrophys. J. 359, 427 (1990). https:// doi.org/10.1086/169075

46. L.G. Gómez, J.A. Rueda, Phys. Rev. D 96, 063001 (2017). https:// doi.org/10.1103/PhysRevD.96.063001

47. A. Caputo, J. Zavala, D. Blas, Phys. Dark Univ. 19, 1-11 (2018). https://doi.org/10.1016/j.dark.2017.10.005. arXiv:1709.03991 [astro-ph.HE] 